\title{
A unified method for signal parameter estimation of arbitrarily distorted single-phase grids with dc-offset
}

\author{
C.M. Hackl ${ }^{\ddagger}, \star$, Senior Member, IEEE and M. Landerer ${ }^{\ddagger}$
}

\begin{abstract}
A unified method is presented which allows to estimate dc-offset, all harmonic components and fundamental frequency in arbitrarily distorted single-phase grids using a Frequency Adaptive Observer (FAO) consisting of modified SecondOrder Generalized Integrators (mSOGIs), a DC-Integrator (DCI) and a modified Frequency Locked Loop (mFLL). DCI and mSOGIs are tuned by pole placement which allows for an arbitrarily fast detection of dc-offset and harmonic components if the fundamental frequency is known. If the fundamental frequency must be estimated as well, a mFLL with Gain Normalization (GN), Rate Limitation (RL), Anti-Windup (AW) strategy and low-pass filters (LPF) must be employed. The effectiveness of the proposed FAO is validated by experimental results and its enhanced performance is shown and compared to existing estimation methods.
\end{abstract}

Index Terms-frequency adaptive observer; second-order generalized integrator; frequency locked loop; frequency estimation; dc-offset estimation, harmonics estimation.

Notation: $\mathbb{N}, \mathbb{R}, \mathbb{C}, \mathbb{Q}$ : natural, real, complex and rational numbers. $\boldsymbol{x}:=\left(x_{1}, \ldots, x_{n}\right)^{\top} \in \mathbb{R}^{n}$ : column vector, $n \in \mathbb{N}$ (where $:=$ means "is defined as" and ${ }^{\top}$ means "transposed"). $\mathbf{0}_{n} \in \mathbb{R}^{n}$ : zero vector. $\|\boldsymbol{x}\|:=\sqrt{\boldsymbol{x}^{\top} \boldsymbol{x}}$ : Euclidean norm of $\boldsymbol{x}$. $\boldsymbol{A} \in \mathbb{R}^{n \times m}$ : real matrix, $n, m \in \mathbb{N}$. $\operatorname{det}(\boldsymbol{A})$ : determinant of A. $\boldsymbol{O}_{n \times m} \in \mathbb{R}^{n \times m}$ : zero matrix. $\bar{z}=\sigma-\jmath \omega \in \mathbb{C}$ : conjugate complex of $z=\sigma+\jmath \omega \in \mathbb{C}$ with $\sigma, \omega \in \mathbb{R}$.

\section{Motivation AND LiteratURE REVIEW}

In future, as large-scale generation systems will be replaced by decentralized energy production like wind or solar, which are coupled to the grid by power electronic devices, the overall grid inertia will diminish. As a consequence, fast(er) frequency fluctuations and (more) distorted voltages and/or currents might occur endangering grid stability and voltage quality which may lead to partial blackouts, the destruction of electronic devices or a threat to human's life. In particular, the presence of dc-offsets will result in malfunctioning grid converters [1]. Additionally, distorted grid quantities yield equivalently distorted powers which then contain, besides dcoffset, harmonic contents with varying frequencies. To take appropriate countermeasures, firstly these distortions must be identified as fast and precise as possible to protect users from malfunctioning equipment and to prevent destruction

${ }^{\ddagger}$ C.M. Hackl is with the Munich University of Applied Sciences (MUAS) and head of the "Laboratory of Mechatronic and Renewable Energy Systems (LMRES)", Germany (e-mail: christoph.hackl@hm.edu).

${ }^{\dagger}$ M. Landerer is with the Munich University of Applied Sciences (MUAS) and research associate at the "Laboratory of Mechatronic and Renewable Energy Systems (LMRES)", Germany (e-mail: markus.landerer@hm.edu).

*Authors are in alphabetical order and contributed equally to the paper Corresponding author is C.M. Hackl (christoph.hackl@hm.edu). of electronic devices. Therefore, in the recent years, much effort has been put into this research question which resulted in the development of Second-Order Generalized Integrators (SOGIs) [2], [3], [4], [5], [6], [7], [8], [9], [10], [11], [12].

These SOGIs rely on an oscillation capability, i.e. they can reduplicate any non-distorted sinusoid (and, as side product, its quadrature signal) with known and constant frequency. For proper functionality, if the frequency is unknown or varying, the SOGIs must be equipped with a Phase Locked Loop [13], [14], [15] or a Frequency Locked Loop [6], [16], [17] to estimate the signal's frequency online.

Hereby, multiple SOGIs with different oscillation frequencies can be parallelized to decompose a distorted input signal into its harmonic components. However, this decomposition of a distorted input signal with multiple harmonics is mostly not considered; only few papers [5], [10], [12], [18], [19], [20], [21] explicitly deal with this topic. In [12], an input signal with six harmonics and varying fundamental frequency was considered; the overall estimation time took about $1,5 \mathrm{~s}$. Additionally, the authors proved the stability of the parallelized SOGI system (without considering the FLL) but did not give a reasonable tuning rule for their observer. [18] used a total of four harmonics and included frequency estimation but also missed to provide guidelines for a good tuning. [19] analyzed up to nine harmonics without considering frequency adaption or tuning parameters; moreover, the implementation of the observer was not thoroughly discussed. In [10], a modification of the parallelized SOGIs was discussed to robustify their performance under frequency fluctuations; however, frequency adaption itself was not considered. Seven harmonics were considered for validation. In [20], a thorough stability analysis of parallelized SOGIs was presented and tuning options were discussed in detail. But, still, the settling time of the estimation algorithm was too long with about $120 \mathrm{~ms}$. Besides frequency adaption and ten harmonics, the presence of a dc-offset was considered in combination with parallelized SOGIs which is, to the best knowledge of the authors, the only publication so far which considers dc-offset and multiple harmonics. All mentioned papers above have in common that they use the so called standard SOGI with solely one gain which only allows for a limited tuning and yields a rather slow estimation performance $(>40-50 \mathrm{~ms})$. In [5], this problem was solved by introducing a so called modified SOGI with two gains which allows "(theoretically) for an arbitrarily fast estimation". Nevertheless, dc-offset was not considered. A different approach was shown in [21], where a new estimation method based on a similarity transformation was introduced with which an overall stability proof was possible. However, 
the authors only considered three harmonic components and did neglect a dc-offset.

In contrast to harmonics estimation, the consideration of dc-offsets in the input signal is more present in the literature; however, the explicit estimation or rejection of the dc-offset is dealt with in rather few publications [1], [22], [23]. A good overview of parameter estimation methods in presence of dc-offset is given in [23], but dc-offset estimation itself is not its focus. All methods described use a standard SOGI as prefilter. Some selected methods are (i) the cascaded SOGI: the first SOGI rejects the dc-offset in its estimated input which therefore can be fed straightforward to the next SOGI; (ii) the DC-SOGI: this method includes, besides the SOGI, a parallel estimation of the dc-offset; and (iii) SOGIs with subsequent calculations like Delayed Signal Cancellations and Complex Coefficient Filters. In [22], several methods aiming at explicit dc-offset estimation are presented. They include (i) a three-phase Phase Locked Loop, (ii) a common DC-SOGI (as in [1], [23]) with frequency adaption and (iii) a transformed DC-SOGI for amplitude-phase and frequency estimation. All methods have in common that (i) they are not designed for harmonics estimation (no parallelized SOGIs) and (ii) they use the slow standard SOGIs with limited tuning capability.

Finally, frequency estimation can be achieved either by Phase Locked Loops (PLLs) [22], [24], [25] or Frequency Locked Loops (FLLs) [16], [26], [27], [28]. In the presence of dc-offsets, the classic frequency adaption is not possible anymore, so in [23] (see above), multiple PLL designs are discussed. In [1], [22], FLLs are considered. The frequency adaption is the bottleneck of the estimation since it significantly decelerates the settling time of the overall estimation process (e.g. [12]: 1,5 s; [20]: $120 \mathrm{~ms}$; [22]: $60 \mathrm{~ms}$ ). Moreover, the presented standard FLLs ( $F F L L s$ ) can get locked at zero frequency or even become unstable. In general, its performance is highly dependent on amplitudes and frequencies of the signals to be analyzed. To encounter these problems, several approaches have been reported this far. One is called gain normalization (GN) [29] and normalizes the frequency adaption in view of signal amplitudes. This approach was further extended by Output Saturation [8], [20] and was finally upgraded to an FLL with GN, sign-correct Anti-Windup (AW) decision function and rate limitation (RL) in [5] to prevent overshooting and achieve a more robust and stable performance.

To the best knowledge of the authors, only one work [20] deals with the estimation of dc-offset, harmonic components and frequency adaption. Unfortunately, the proposed approach has rather slow settling times and implementation is rather complicated.

That is why this article proposes an overall estimation system - the Frequency Adaptive Observer (FAO) - which is capable of estimating (i) dc-offset, (ii) fundamental frequency and (iii) harmonic components with fast estimation performance and simple implementation. Moreover, for known frequencies, the FAO can be tuned such that it can estimate all parameters with a prescribed settling time. Concluding, the contributions of this paper are extensions of [5] and can be summarized as follows: (i) Introduction of a generic and parallelized observer structure consisting of DC-Integrator (DCI) and modified Second-Order Generalized Integrators (mSOGIs) and the analytical computation of the observer gains which achieves prescribed settling time by pole placement (see Section III-D);

(ii) Extensions of the modified Frequency Locked Loop ( $m F L L)$ with low-pass filters, gain normalization, antiwindup and rate limitation for proper functionality in combination with the parallelized DCI+mSOGIs leading to the overall FAO (see Section III-E); and

(iii) Implementation and experimental validation of the proposed parallelized DCI+mSOGIs and the overall FAO in comparison to the existing parallelized sSOGI+sFLL system without dc-offset estimation and a non-parallelized DC+sSOGI+sFLL system (see Section IV).

\section{Problem Statement}

Single-phase grid signals with arbitrarily many harmonic components and dc-offset are considered and described by

$$
\forall t \geq 0: \quad y(t):=a_{0}(t)+\sum_{\nu \in \mathbb{H}_{\nu}} \underbrace{a_{\nu}(t) \cos \left(\phi_{\nu}(t)\right)}_{:=y_{\nu}(t)},
$$

where $\mathbb{H}_{\nu}:=\left\{\nu_{1}, \nu_{2}, \ldots, \nu_{n}\right\} \subset \mathbb{Q}_{>0}$ is the set of all considered (known) harmonic orders (with fundamental order $\nu_{1}=1$ ). Moreover, the harmonic phase angles

$$
\forall \nu \in \mathbb{H}_{\nu} \forall t \geq 0: \quad \phi_{\nu}(t):=\int_{0}^{t} \nu \omega(\tau) \mathrm{d} \tau+\phi_{\nu, 0}(t)
$$

depend on the possibly time-varying ${ }^{1}$ angular frequency $\omega>$ $0 \frac{\mathrm{rad}}{\mathrm{s}}$; also, the dc-offset $a_{0}$, the amplitudes $a_{\nu}$ and angles $\phi_{\nu, 0}$ are allowed to be time-varying. Goal is to estimate $y$ as in (1) by its estimate (indicated by "へ")

$$
\forall t \geq 0: \quad \widehat{y}(t):=\widehat{a}_{0}(t)+\sum_{\nu \in \mathbb{H}_{\nu}} \underbrace{\widehat{a}_{\nu}(t) \cos \left(\widehat{\phi}_{\nu}(t)\right)}_{:=\widehat{y}_{\nu}(t)},
$$

which shall be decomposed into the estimates of the harmonic components $\widehat{y}_{\nu}$ and the dc-offset $\widehat{a}_{0}$, respectively. This estimation requires the estimate $\widehat{\omega}$ of the fundamental angular frequency. To achieve this goal, this paper proposes (i) the parallelization of a DC-integrator (DCI) and several modified SOGIs (mSOGIs) and (ii) the use of a modified Frequency Locked Loop (mFLL) with Gain Normalization (GN), signcorrect Anti-Windup (AW), Rate Limitation (RL) and low-pass filters LPFs. Both lead to the overall FAO for a fast and robust estimation of all crucial grid signal parameters in real time.

\section{Proposed solution: Frequency AdAptive OBSERVER (FAO)}

This section presents the theoretical background for the development and implementation of the FAO. It consists of the parallelization of mSOGIs (as introduced in [5]) and a DCI. The principle idea of the FAO is based on the Internal Model Principle (as introduced by Wonham in the 1980s [30]). The

\footnotetext{
${ }^{1}$ The common representation $\phi_{\nu}=\nu \omega$ is an unnecessary simplification.
} 
internal model principle states that any observation problem can be solved by a generating system being (i) capable of reduplicating its input signal and (ii) equipped with feedback.

\section{A. Generating system (Internal Model Principle)}

Note that any constant (such as the dc-offset $a_{0}$ ) can be generated by the following integrator

$$
\frac{\mathrm{d}}{\mathrm{d} t} x_{0}(t)=0 \cdot x_{0}(t), \quad x_{0}(0)=x_{0,0}, \quad a_{0}(t)=1 \cdot x_{0}(t),
$$

with zero input and appropriate initial value $x_{0,0}$. Moreover, any sinusoidal signal $y_{\nu}$ (e.g. the $\nu$-th harmonic component) and its respective quadrature signal $q_{\nu}$ can be reduplicated by the following second-order dynamical system

$$
\left.\begin{array}{rl}
\frac{\mathrm{d}}{\mathrm{d} t\left(\begin{array}{c}
x_{\nu}(t) \\
q_{\nu}(t)
\end{array}\right)} & =\nu \omega \overbrace{\left[\begin{array}{cc}
0 & -1 \\
1 & 0
\end{array}\right]}^{\boldsymbol{x}_{\nu}(t) \in \mathbb{R}^{2}} \boldsymbol{x}_{\nu}(t), \quad \boldsymbol{x}_{\nu}(0)=\boldsymbol{x}_{\nu, 0}, \\
y_{\nu}(t) & =\underbrace{\left(\begin{array}{ll}
1, & 0
\end{array}\right)}_{=: \boldsymbol{c}_{\nu}^{\top} \in \mathbb{R}^{2}} \boldsymbol{x}_{\nu}(t),
\end{array}\right\}
$$

which represents an harmonic oscillator. Amplitude $a_{\nu}$ and phase angle $\phi_{\nu}$ are determined by the initial values in $\boldsymbol{x}_{\nu, 0}$. The overall generating system, which is able to reduplicate $y$ as in (1), consists of the parallelization of (3) and (4). Hence, its dynamics are given by (cf. [5]; there without $x_{0}$ )

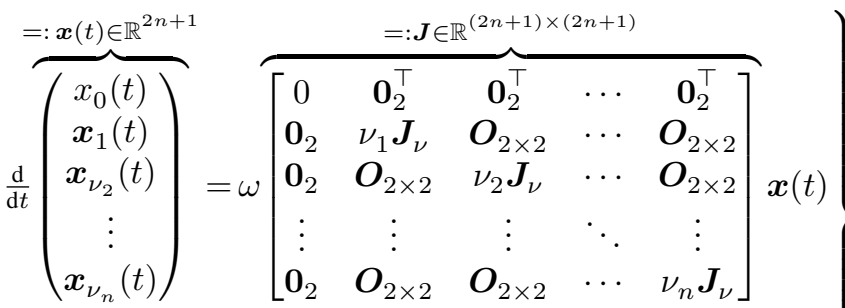

$$
\begin{aligned}
& y(t)=\underbrace{\left(1, \boldsymbol{c}_{\nu}^{\top}, \cdots, \boldsymbol{c}_{\nu}^{\top}\right)}_{=: \boldsymbol{c}^{\top} \in \mathbb{R}^{2 n+1}} \boldsymbol{x}(t) .
\end{aligned}
$$

If all initial values in $\boldsymbol{x}(0)=\boldsymbol{x}_{0}$ and the angular frequency $\omega$ were known, then (5) could perfectly reduplicate the singlephase signal $y$ as in (1). But, since those values are not known a priori, an observer must be designed which is actually feasible since the dynamics (5) are fully state observable, i.e.

$$
\operatorname{rank}\left[\boldsymbol{c}, \quad \boldsymbol{J}^{\top} \boldsymbol{c}, \quad \cdots, \quad\left(\boldsymbol{J}^{\top}\right)^{2 n} \boldsymbol{c}\right]^{\top}=2 n+1 ;
$$

as a straight forward extension of the proof in [31] shows.

\section{B. DC-Integrator $(D C I)$}

To estimate the dc-offset online, the generating system (3) is equipped with the feedback $-\widehat{\omega}(t) l_{0}\left(y_{0}(t)-\widehat{y}_{0}(t)\right)$ which leads to the DC-Integrator (DCI) dynamics

$$
\left.\begin{array}{rl}
\frac{\mathrm{d}}{\mathrm{d} t} \widehat{x}_{0}(t) & =-\widehat{\omega}(t) l_{0} \widehat{x}_{0}(t)+\widehat{\omega}(t) l_{0} y_{0}(t), \quad \widehat{x}_{0}(0)=0 \\
\widehat{y}_{0}(t) & =\widehat{x}_{0}(t)
\end{array}\right\}
$$

which is a simple first-order system (see Fig. 1(a)) which achieves $\widehat{y}_{0} \rightarrow y_{0}$ and can be arbitrarily tuned by the gain $l_{0}>0$. The estimated angular frequency $\widehat{\omega}(t)$ scales the system dynamics and is obtained by the modified Frequency Locked Loop (mFLL; see Sec. III-E). Note that the real dcoffset $y_{0}$ is actually not available for feedback, only the signal $y$ as in (1) is measured. This fact leads to the parallelization in Sec. III-D.

\section{Modified Second-Order Generalized Integrator (mSOGI)}

To estimate the $\nu$-th sinusoidal-like harmonics, the internal model in (4) is used with $\widehat{\omega}$ instead of $\omega$ and with the feedback

$$
\widehat{\omega}(t) \underbrace{\left(\begin{array}{c}
k_{\nu} \\
g_{\nu}
\end{array}\right)}_{=: \boldsymbol{l}_{\nu}}(y_{\nu}(t)-\underbrace{\left(\begin{array}{ll}
1, & 0
\end{array}\right)}_{=: \boldsymbol{c}_{\nu}^{\top}} \underbrace{\left(\begin{array}{c}
\widehat{y}_{\nu}(t) \\
\widehat{q}_{\nu}(t)
\end{array}\right)}_{=: \widehat{\boldsymbol{x}}_{\nu}(t)}),
$$

which yields the mSOGI dynamics (for details see [5] and Fig. 1(b))

$$
\left.\begin{array}{rl}
\frac{\mathrm{d}}{\mathrm{d} t} \widehat{\boldsymbol{x}}_{\nu}(t) & =\widehat{\omega}(t)\left[\nu \boldsymbol{J}_{\nu}-\boldsymbol{l}_{\nu} \boldsymbol{c}_{\nu}^{\top}\right] \widehat{\boldsymbol{x}}(t)+\widehat{\omega}(t) \boldsymbol{l}_{\nu} y_{\nu}(t) \\
\widehat{y}_{\nu}(t) & =\boldsymbol{c}_{\nu}^{\top} \widehat{\boldsymbol{x}}_{\nu}(t) .
\end{array}\right\}
$$

The purpose of the mSOGI - similar to the standard SOGI (sSOGI) with $g_{\nu}=0$ in (7), cf. [5], [29] - is to estimate a given sinusoidal signal $y_{\nu}$ and its quadrature signal $\widehat{q}$ such that $\widehat{y}_{\nu} \rightarrow y_{\nu}$ and $\widehat{q}_{\nu} \rightarrow q_{\nu}$. Its working principle is based on the oscillating nature of its dynamics whose resonance frequency is the harmonic angular frequency $\widehat{\omega}$. Note that the mSOGI compared to the sSOGI achieves a (much) faster estimation, since its poles can be arbitrarily chosen as now the two feedback gains $k_{\nu}$ and $g_{\nu}$ are available for tuning instead of only one feedback gain $k_{\nu}$ (and $g_{\nu}=0$ ) as for the sSOGI [29]. Similar to the DCI, feedback of $y_{\nu}$ is not feasible, as only $y$ as in (1) is available for feedback which motivates for the parallelization of DCI and mSOGI as discussed next.

\section{Parallelization of DCI and mSOGIs}

As already noted, the problem is that the sub-signals $y_{0}$ and $y_{\nu}$ are not available for feedback. Only the overall signal $y$ as in (1) is measured and can be fed to the overall observer. Hence, a parallelized structure must be set up (see Fig. 4).

To do so, for dc-offset and each harmonic order $\nu \in \mathbb{H}_{\nu}$, DCI (6) and each $\nu$-th mSOGI (7) are merged and parallelized leading to the overall observer dynamics

$$
\left.\begin{array}{rl}
\frac{\mathrm{d}}{\mathrm{d} t} \widehat{\boldsymbol{x}}(t) & =\widehat{\omega}(t)\left[\boldsymbol{J}-\boldsymbol{l} \boldsymbol{c}^{\top}\right] \widehat{\boldsymbol{x}}(t)+\widehat{\omega}(t) \boldsymbol{l} y(t), \widehat{\boldsymbol{x}}(0)=\widehat{\boldsymbol{x}}_{0}, \\
\widehat{y}(t) & =\boldsymbol{c}^{\top} \widehat{\boldsymbol{x}}(t)
\end{array}\right\}
$$

where $\widehat{\boldsymbol{x}}:=\left(\widehat{x}_{0}, \widehat{y}_{\nu_{1}}, \widehat{q}_{\nu_{1}}, \cdots, \widehat{y}_{\nu_{n}}, \widehat{q}_{\nu_{n}}\right)^{\top} \in \mathbb{R}^{2 n+1}$ and $\boldsymbol{l}:=$ $\left(l_{0}, k_{\nu_{1}}, g_{\nu_{1}}, \cdots, k_{\nu_{n}}, g_{\nu_{n}}\right)^{\top} \in \mathbb{R}^{2 n+1}$ consist of all substates and sub-feedback gains of DCI and mSOGIs, respectively. Clearly, in (8), only the available measured signal $y$ is used for feedback. Note that this generic observer structure for dc-offset and arbitrary harmonics estimation has not yet been reported in literature. In [1], [23], the trivial observer structure for dc-offset and fundamental signal estimation (i.e. $n=1$ ) has been discussed.

Furthermore, note that, for a parallelized sSOGI design (with $g_{\nu}=0$ for all $\nu \in \mathbb{H}_{\nu}$ ), the feedback vector $\boldsymbol{l}=$ $\left(l_{0}, k_{\nu_{1}}, 0, \cdots, k_{\nu_{n}}, 0\right)^{\top}$ has only $n+1$ gains whereas the 


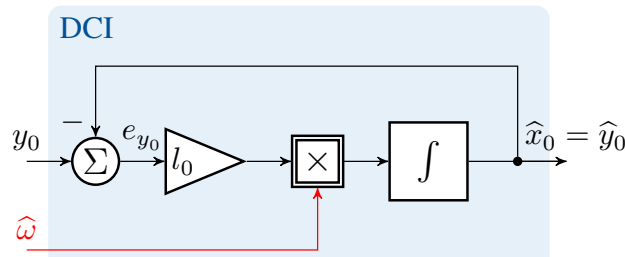

(a) Block diagram of DC-integrator (DCI).

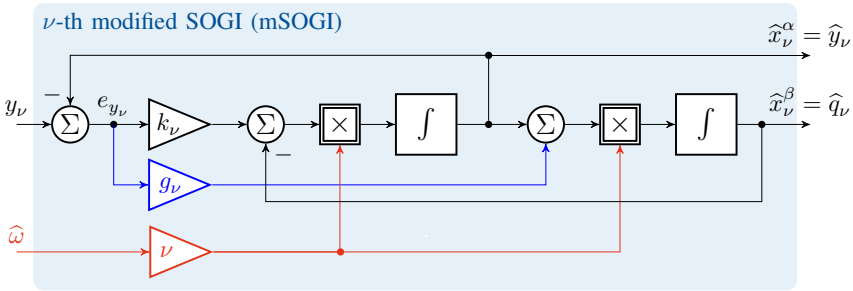

(b) Block diagram of $\nu$-th modified Second-order Generalized Integrator (mSOGI) [5].

Figure 1: Components of the Frequency Adaptive Observer (FAO): (a) DC-Integrator (DCI) and (b) modified SOGI (mSOGI).

obverser matrix $\boldsymbol{J}-\boldsymbol{l} \boldsymbol{c}^{\top}$ has still $2 n+1$ eigenvalues (poles). Hence, solely the observer with parallelized mSOGIs allows for an arbitrary tuning by pole placement (cf. [5]). Pole placement can be easily achieved by MATLAB which provides the place command, i.e.

$$
\boldsymbol{l}^{\top}=\operatorname{place}\left(\boldsymbol{J}^{\top}, \boldsymbol{c}, \boldsymbol{p}^{\star}\right),
$$

which allows to assign the (desired) eigenvalues $p^{\star}:=$ $\left(p_{0}^{\star}, p_{\nu_{1}}^{\star}, \bar{p}_{\nu_{1}}^{\star}, \ldots, p_{\nu_{n}}^{\star}, \bar{p}_{\nu_{n}}^{\star}\right)^{\top} \in \mathbb{R}^{2 n+1}$ of the observer matrix $\boldsymbol{J}-\boldsymbol{l} \boldsymbol{c}^{\top}$. The vector $\boldsymbol{p}^{\star}$ contains the desired poles $p_{0}^{*} \in \mathbb{R}$ of the DCI and the desired poles $p_{\nu}^{\star}, \bar{p}_{\nu}^{\star} \in \mathbb{C}$ of the parallelized mSOGIs for all $\nu \in\left\{\nu_{1}, \ldots, \nu_{n}\right\}$. However, this command requires that the multiplicity of the poles is not greater than $\operatorname{rank}(\boldsymbol{c})=1$. If, for some reason, this is desired, one could take advantage of the following analytic gain calculation

$$
\boldsymbol{l}=\left[\begin{array}{cc}
\mathbf{0}_{2 n}^{\top} & \frac{1}{\prod_{\nu \in \mathbb{H}_{n}} \nu^{2}} \\
\boldsymbol{S} & -\frac{1}{\prod_{\nu \in \mathbb{H}_{n}} \nu^{2}} \boldsymbol{S w}
\end{array}\right] \widetilde{\boldsymbol{p}}_{\boldsymbol{A}}^{\star}
$$

with $\quad \boldsymbol{R}_{i}:=\left[\begin{array}{cc}1 & 0 \\ 0 & -\frac{1}{\nu_{i}}\end{array}\right], \quad \boldsymbol{S}:=\left[\begin{array}{ccc}S_{1,1} & \cdots & s_{n, 1} \\ \vdots & \ddots & \vdots \\ s_{1, n} & \cdots & s_{n, n}\end{array}\right]$, $\boldsymbol{S}_{c, r}:=(-1)^{c+1} \nu_{r}^{2(n-c)} \boldsymbol{R}_{r} \prod_{\substack{i=1 \\ i \neq r}}\left(\nu_{r}^{2}-\nu_{i}^{2}\right)^{-1}, \boldsymbol{w}:=$ $\left(1,0, \sum_{\nu \in \mathbb{H}_{n}} \nu^{2}, 0, \ldots, \sum_{\nu \in \mathbb{H}_{n}} \prod_{\substack{\mu \in \mathbb{H}_{n} \\ \mu \neq \nu}} \mu^{2}, 0\right)^{\top}$ and $\widetilde{\boldsymbol{p}}_{\boldsymbol{A}}^{\star}:=$ $\left(-\sum_{i=1}^{2 n+1} p_{i}^{\star}, \sum_{i=1}^{2 n+1} p_{i}^{\star} \sum_{j=i+1}^{2 n+1} p_{j}^{\star}-\sum_{\nu \in \mathbb{H}_{n}} \nu^{2}, \ldots, \sum_{i=1}^{2 n+1} \prod_{\substack{j=1 \\ j \neq i}}^{2 n+1} p_{j}^{\star}-\right.$ $\left.\prod_{\nu \in \mathbb{H}_{n}} \nu^{2},-\prod_{i=1}^{2 n+1} p_{i}^{\star}\right)^{\top}$ which is an extension of Eq. (15) in [31]. A good tuning is achieved by the choices $p_{0}^{\star} \leq$ $\min _{\nu} \Re\left(p_{\nu}^{\star}\right)$ and $p_{\nu}^{\star}=-\sigma^{\star}+\jmath \nu^{\star}$ with $\sigma^{\star}>0$ (e.g. $\sigma^{\star}=\frac{3}{2}$ or 2) and $\nu^{\star}=\nu \in \mathbb{H}_{\nu}$ to preserve a (damped) oscillating behavior of the mSOGIs. Since the eigenvalue(s) or pole(s) closest to the imaginary axis (i.e. those poles with $\max _{\nu} \Re\left(p_{i}^{\star}\right)$ ) determine the overall settling time of the parallelized DCImSOGIs system, the minimum settling time can (theoretically) be specified arbitrarily. However, noise sensitivity and overshooting may limit the achievable response; in particular since the overall poles of the varying matrix $\widehat{\omega}(t)\left[\boldsymbol{J}-\boldsymbol{l} \boldsymbol{c}^{\top}\right]$ are scaled by the frequency estimate $\widehat{\omega}(t)$.

\section{E. Frequency adaption by modified FLL (mFLL)}

The last ingredient of the FAO is the estimation or - more precisely - the adaption of the angular frequency by the modified frequency locked loop (mFLL). Goal is to achieve asymptotic adaption of $\widehat{\omega}(t)$ such that $\widehat{\omega} \rightarrow \omega$. The principle adaption law

$$
\frac{\mathrm{d}}{\mathrm{d} t} \widehat{\omega}(t) \propto \gamma(t)(\underbrace{y(t)-\widehat{y}(t)}_{=: e_{y}(t)}) \boldsymbol{\lambda}^{\top} \widehat{\boldsymbol{x}}(t), \quad \widehat{\omega}(0)=\widehat{\omega}_{0}
$$

of the mFLL is based on a steady-state analysis of the parallelized DCI and mSOGIs (similar to the analysis in [31]). The selection vector $\boldsymbol{\lambda}:=\left(0, g_{\nu_{1}},-k_{\nu_{1}}, 0, \cdots, 0\right)^{\top} \in \mathbb{R}^{2 n+1}$ extracts only the fundamental components of the estimated in-phase signal $\widehat{y}_{\nu_{1}}$ and its quadrature signal $\widehat{q}_{\nu_{1}}$ such that the product $e_{y} \boldsymbol{\lambda}^{\top} \widehat{\boldsymbol{x}}=e_{y} \boldsymbol{l}_{\nu_{1}}^{\top} \boldsymbol{J}_{\nu} \widehat{\boldsymbol{x}}_{\nu_{1}}$ is in-phase with the input estimation error $e_{y}=y-\widehat{y}$. Observe the inverse weighting of $\widehat{y}_{\nu_{1}}$ by $g_{\nu_{1}}$ and $\widehat{q}_{\nu_{1}}$ by $-k_{\nu_{1}}$. The adaption in (11) achieves (on average over one fundamental period) a sign-correct adaption of the angular frequency estimate $\widehat{\omega}$ for all positive gains $\gamma(t)>0$. The initial value $\widehat{\omega}_{0}$ helps to improve the transient behavior of the mFLL; e.g. in Europe, the nominal frequency is $f_{0}=50 \mathrm{~Hz}$, therefore the initial (nominal) value should be $\omega_{0}=2 \pi f_{0}$.

As proposed in [5], the principle adaption law (11) should be extended by (i) a gain normalization (GN), (ii) a sign-correct anti-windup (AW) decision function

$$
f_{\text {aw }}(\widehat{\omega}, \delta):=\left\{\begin{array}{rr}
0, & \text { for }\left(\widehat{\omega} \geq \omega_{\max } \wedge \delta \propto \frac{\mathrm{d}}{\mathrm{d} t} \widehat{\omega} \geq 0\right) \\
& \vee\left(\widehat{\omega} \leq \omega_{\min } \wedge \delta \propto \frac{\mathrm{d}}{\mathrm{d} t} \widehat{\omega} \leq 0\right) \\
1, & \text { else }
\end{array}\right.
$$

and (iii) a rate limitation (RL)

$$
\operatorname{sat}_{\dot{\omega}_{\min }}^{\dot{\omega}_{\max }}[\delta]:=\left\{\begin{array}{l}
\dot{\omega}_{\max }, \delta>\dot{\omega}_{\max } \\
\delta, \dot{\omega}_{\min } \leq \delta \leq \dot{\omega}_{\max } \\
\dot{\omega}_{\min }, \delta<\dot{\omega}_{\min }
\end{array}\right.
$$

to achieve a stable and smooth(er) frequency estimation. Moreover, in view of measurement noise, additional low-pass filters (LPFs) should be included in the mFLL. The considered LPFs (see Fig. 2) have cut-off frequency $\omega_{c}$ and the following first-order dynamics

$$
\frac{\mathrm{d}}{\mathrm{d} t} \xi^{\mathrm{lpf}}(t)=-\omega_{c} \xi^{\mathrm{lpf}}(t)+\omega_{c} \xi(t), \quad \xi^{\mathrm{lpf}}(0)=0 .
$$

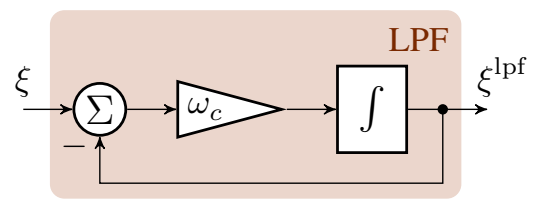

Figure 2: Block diagram of the low-pass filters (LPFs) used in the mFLL. 
Three LPFs should be implemented for the three signals $e_{y}$, $\widehat{y}_{\nu_{1}}$ and $\widehat{q}_{\nu_{1}}$ to achieve synchronicity (identical phase lag) of all filtered signals $e_{y}^{\operatorname{lpf}}, \widehat{y}_{\nu_{1}}^{\operatorname{lpf}}$ and $\widehat{q}_{\nu_{1}}^{\mathrm{lpf}}$, respectively. Bringing all together, finally, leads to the modified frequency locked loop (mFLL) as illustrated in Fig. 3 with the overall adaption law

$$
\frac{\mathrm{d}}{\mathrm{d} t} \widehat{\omega}(t)=f_{\mathrm{aw}}(\widehat{\omega}(t), \delta(t)) \operatorname{sat}_{\dot{\omega}_{\min }}^{\dot{\dot{\omega}}_{\max }}[\overbrace{\left.\frac{\Gamma \widehat{\omega}(t) e_{y}^{\mathrm{pp}}(t) \boldsymbol{l}_{\nu_{1}}^{\top} \boldsymbol{J}_{\nu} \widehat{\boldsymbol{x}}_{\nu_{1}}^{\mathrm{ppf}}(t)}{\max \left(\left\|\widehat{\boldsymbol{x}}_{\nu_{1}}^{\mathrm{pf}}(t)\right\|^{2}, \varepsilon\right)}\right]}^{=: \delta(t)}]
$$

with some constant gain $\Gamma>0$. In these functions, the upper and lower limits $\omega_{\max }, \omega_{\min }, \dot{\omega}_{\max }$ and $\dot{\omega}_{\min }$ guarantee that the frequency estimate $\widehat{\omega}$ is (i) always bounded away from zero (crucial for stability of the FAO, cf. [5]) and stays within the interval $\left[\omega_{\min }, \omega_{\max }\right]$ and (ii) the adaption is not too fast yielding a more robust adaption (for details see [5]). Concluding, note that the standard FLL (sFLL) [29], which is usually implemented, comes only with GN but without AW, RL and LPFs.

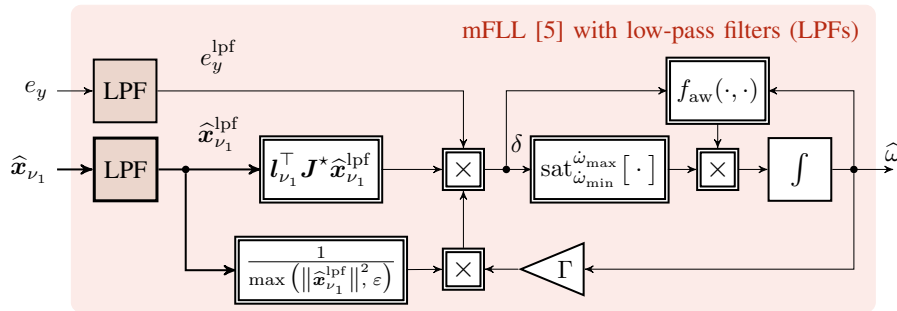

Figure 3: Block diagram of modified Frequency Locked Loop (mFLL) with gain normalization (GN), sign-correct anti-windup (AW) decision function, rate limitation (RL) and low-pass filters (LPFs).

\section{F. Overall structure of FAO}

The block diagram of the complete FAO, consisting of parallelized DCI (6) and mSOGIs (7), and mFLL (15) is illustrated in Fig. 4. The FAO is fed by the input $y$ as in (1) and provides the estimates $\widehat{y}$ as in (2) and $\widehat{\omega}$ (according to adaption as in (15)), and the overall estimation state vector $\widehat{\boldsymbol{x}}=\left(\widehat{x}_{0}, \widehat{y}_{\nu_{1}}, \widehat{q}_{\nu_{1}}, \cdots, \widehat{y}_{\nu_{n}}, \widehat{q}_{\nu_{n}}\right)^{\top}$, which contains the (i) dcoffset estimate $\widehat{a}_{0}=\widehat{x}_{0}$ and, for $\nu \in \mathbb{H}_{\nu}$, (ii) all harmonic in-phase $\widehat{y}_{\nu}$ and quadrature $\widehat{q}_{\nu}$ estimates. Based on the estimated individual harmonic sub-state vector $\widehat{\boldsymbol{x}}_{\nu}=\left(\widehat{y}_{\nu}, \widehat{q}_{\nu}\right)^{\top}$, amplitude estimate $\widehat{a}_{\nu}:=\left\|\widehat{\boldsymbol{x}}_{\nu}\right\|$ and phase angle estimate $\widehat{\phi}_{\nu}:=\arctan 2\left(\widehat{y}_{\nu}, \widehat{q}_{\nu}\right)$ of each harmonic component can be computed for all $\nu \in \mathbb{H}_{\nu}$. Note that the mFLL provides the angular frequency estimate $\widehat{\omega}$ to DCI and all mSOGIs.

\section{IMPLEMENTATION AND MEASUREMENT RESULTS}

To verify the proposed FAO, experimental results are carried out where three estimation methods and their estimation performance are compared to each other. The proposed FAO consisting of DCI, n parallelized mSOGIs and $m F L L$ (in the following labeled as $\mathbf{m F A O _ { 0 , n }}$ ) is compared to the only two standard approaches for dc-offset and harmonics estimation available in literature this far. The available methods are (i) a simple FAO (labeled as $\mathbf{s F A O _ { 0 , 1 }}$ ) consisting of a dc-offset estimator (similar to DCI), one sSOGI (for fundamental signal only) and $s F L L$ as proposed in [1] and (ii) a parallelization of

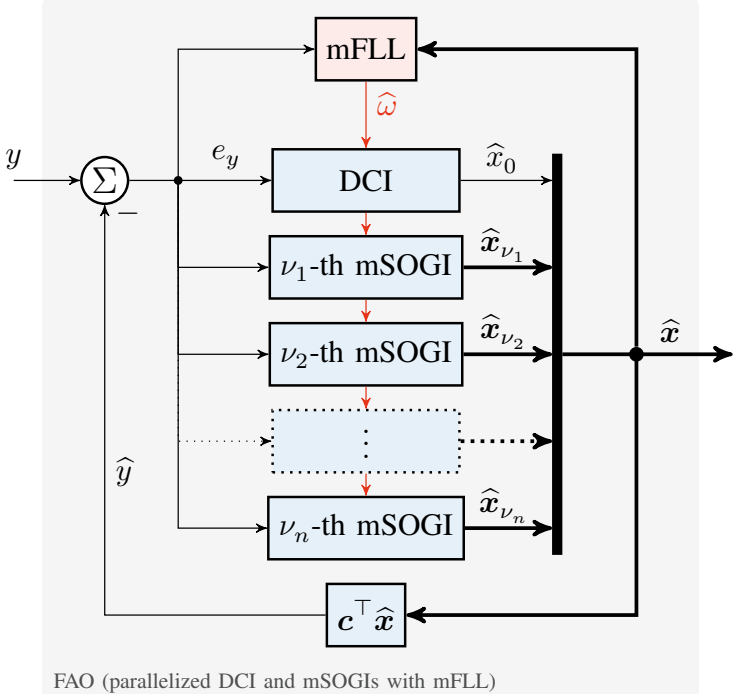

Figure 4: Block diagram of the Frequency Adaptive Observer (FAO).

$n$ sSOGIs with sFLL but without DCI (labeled as $\mathbf{S F A O} \emptyset_{\emptyset, n}$ ) as proposed in [18]. For the experiments, the signal to be investigated is generated in Matlab/Simulink R2018b by the internal model (5), which is downloaded via LAN to the dSPACE Processor Board DS1007 where the signal is produced in real time, converted from digital to analogue by the dSPACE I/O card DS2103, amplified by a Spitzenberger Spies PAS 5000 four quadrant amplifier, measured by a LEM CV 3-1000 voltage sensor and converted from analogue to digital by the dSPACE I/O card DS2004 and, finally, processed by all three estimation methods described above in real time. After the experiment, the measured (recorded) input signal and estimated quantities are analyzed and recorded on a HostPC. The dSPACE system and the amplifier are connected via a ten meters long BNC cable. Four scenarios are discussed:

(S1): For the first scenario, one (fundamental) sinusoidal signal with dc-offset and known frequency is considered. Since frequency is known, all three estimation methods are implemented without FLL. The considered signal undergoes a dc-offset jump of $+100 \mathrm{~V}$ at $t=0,12 \mathrm{~s}$, an amplitude sag of $-75 \%$ at $t=0,24 \mathrm{~s}$, a phase jump of $-\frac{\pi}{2} \mathrm{rad}$ at $t=0,36 \mathrm{~s}$ and reverse jumps in all changed parameters at $t=0,48 \mathrm{~s}$ (see Figs. 5 and 6).

(S2): For the second scenario, an input signal consisting of dc-offset, fundamental and nine harmonics with known frequency is chosen and fed to all three estimation methods. Since frequency is known, the FLLs are not required and de-activated. The step-like signal parameter changes are identical to Scenario (S1). The results are shown in Figs. 7 and 8.

(S3): The third scenario again considers only one (fundamental) sinusoidal signal with dc-offset but with unknown and varying frequency. Hence, all estimation methods require a FLL to estimate the frequency. The considered signal undergoes frequency jumps of $+2 \pi 10 \frac{\mathrm{rad}}{\mathrm{s}}$ at $t=0,12 \mathrm{~s}$ and is shifted about $+\frac{\pi}{2} \mathrm{rad}$ at $t=0,24 \mathrm{~s}$; at $t=0,36 \mathrm{~s}$, an error is emulated where the fundamental signal is nullified and only the dc-offset remains. Finally, at $t=0,48 \mathrm{~s}$, all 
signal parameter changes are reversed (see Figs. 9 and $10)$.

(S4): The last scenario uses the signal from Scenario (S2) containing dc-offset, fundamental and nine harmonics, but this time, with unknown and varying frequency including frequency jumps of $+2 \pi 10 \frac{\mathrm{rad}}{\mathrm{s}}$ at $t=0,12 \mathrm{~s}$ and $-2 \pi 10 \frac{\mathrm{rad}}{\mathrm{s}}$ at $t=0,48 \mathrm{~s}$. Hence, all estimation methods require a FLL to work properly. The other signal parameter changes are identical to those in Scenario (S3) but, now, all harmonic components are nullified such that only the dc-offset remains for a certain time interval. The estimation results are illustrated in Figs. 11 and 12.

For Scenarios (S1) and (S3), the initial signal parameters (at $t=0$ ) are as follows: $a_{0}=-50 \mathrm{~V}, a_{1}=200 \mathrm{~V}, \phi_{1}=0 \mathrm{rad}$ and $\omega=2 \pi 50 \frac{\mathrm{rad}}{\mathrm{s}}$. For Scenarios (S2) and (S4), the respective signal parameters are collected in Tab. I. Considering the system parameters, the gains of $\mathbf{s F A O}{ }_{0,1}$ are taken from [1] and the ones of $\mathbf{S F A O}_{\emptyset, n}$ are copied from [18]. For the proposed $\mathbf{m F A O}_{0, n}$, pole placement as in (10) is performed such that all poles and eigenvalues of $\boldsymbol{J}$ are shifted by -2 into the negative half plane which is a reasonable compromise between noise sensitivity, overshooting and estimation speed. All system parameters are listed in Tab. II.

Table I: Initial signal parameters (at $t=0$ ) for Scenarios (S2) and (S4)

\begin{tabular}{l|ccccccccccc}
\hline$\nu$ & 0 & 1 & 2 & 3 & 4 & 5 & 6 & 7 & 8 & 9 & 10 \\
\hline$a_{\nu} / \mathrm{V}$ & -50 & 200 & 80 & 40 & 120 & 0 & 80 & 0 & 120 & 40 & 40 \\
$\phi_{\nu} / \mathrm{rad}$ & & 0 & $\frac{\pi}{2}$ & $\frac{3 \pi}{2}$ & 0 & $\frac{2 \pi}{3}$ & $\frac{\pi}{4}$ & 0 & $\frac{5 \pi}{4}$ & $\frac{5 \pi}{3}$ & 0 \\
$\omega / \frac{\mathrm{rad}}{\mathrm{s}}$ & & & & & $2 \pi 50$ & & & & & \\
\hline
\end{tabular}

Table II: System parameters of all estimation methods for the experiments.

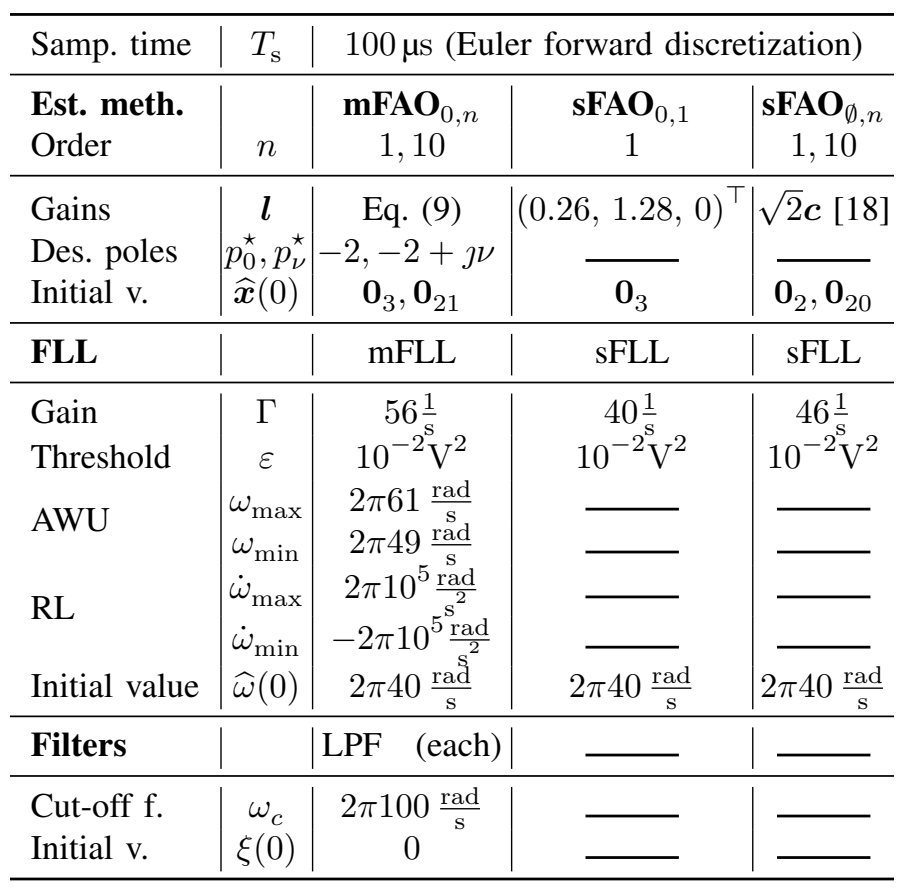

\section{A. Discussion of Scenario (S1)}

For Scenario (S1), a signal with dc-offset, fundamental component (only) and known frequency with step-like changes in the signal parameters is fed to the three FAOs without using the FLLs (which is still implemented but adaption is turned off; its initial value is set to the known angular frequency, i.e. $\widehat{\omega}=\omega \Rightarrow \widehat{f}=f)$. All three estimation methods, i.e. $\mathbf{m F A O}_{0, n}, \mathbf{s F A O}_{0,1}$ and $\mathbf{S F A O} \mathbf{O}_{\emptyset, n}$, are implemented with only one SOGI (i.e. $n=1$ ).

In Fig. 5, the measurement results for Scenario (S1) are shown. The first subplot shows input $y(---)$ and estimation output $\hat{y}$ of $\mathbf{s F A O}_{0,1}(-), \mathbf{S F A O}_{\emptyset, n}(-)$ and $\mathbf{m F A O}_{0, n}$ (-), respectively. In the second subplot, the respective estimation errors $e_{y}=y-\widehat{y}$ are plotted. Due to the choice of the poles, the estimation error of the $\mathbf{m F A O}_{0, n}$ decays significantly faster. Estimation is accomplished within a few milliseconds. In contrast to that, the $\mathbf{S F A O}_{0,1}$ needs about $40-50 \mathrm{~ms}$ to achieve good estimation and the $\mathbf{S F A O} \mathbf{\emptyset}_{\emptyset, n}$ is not capable of estimating the input at all in view of the dc-offset present.
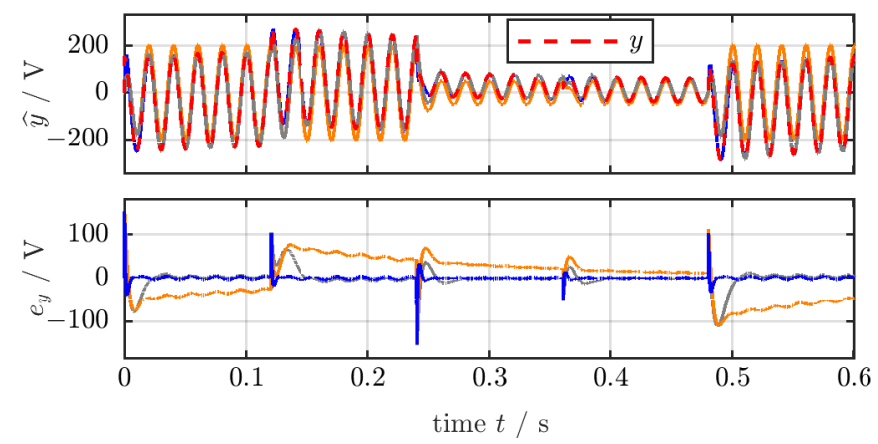

Figure 5: Measurement results for Scenario (S1) (fundamental signal with dc offset and known frequency): The signals shown are input $y(--)$, estimate $\widehat{y}$ and estimation error $e_{y}=y-\widehat{y}$ of $\mathbf{s F A O}_{0,1}(-)$, $\mathbf{S F A O}_{\emptyset, n}(-)$ and $\mathbf{m F A O} \mathbf{O}_{0, n}\left(-\frac{}{-}\right)$, respectively.

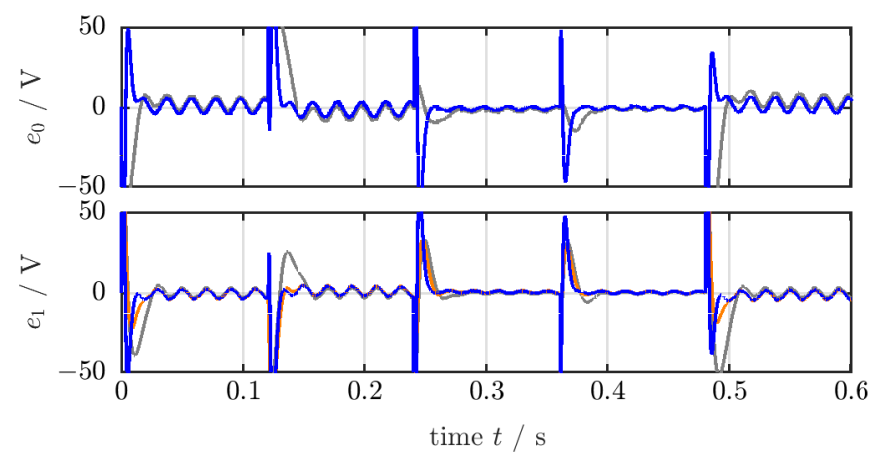

Figure 6: Zoomed-in estimation errors of dc-offset and harmonic signal for Scenario (S1) (fundamental signal with dc-offset and known frequency): The signals shown are estimation errors $e_{0}=y_{0}-\widehat{y}_{0}$ and $e_{1}=y_{1}-\widehat{y}_{1}$ of $\mathbf{s F A O}_{0,1}(-), \mathbf{s F A O}_{\emptyset, n}(-)$ and $\mathbf{m F A O}_{0, n}$ $(-)$, respectively.

Note that, in view of the length of the BNC cable connecting amplifier and dSPACE system, the cable acts as a high-pass filter with rather large time constant such that any dc-offset jump is damped and decays slowly within the considered time intervals. Nevertheless, note that $\mathbf{S F A O} \mathbf{O}_{0,1}$ and $\mathbf{m F A O}_{0, n}$ can track this decaying dc-offset asymptotically. The minor oscillations in all estimation responses are due to the fixed sampling frequency which yields a time lag between $y$ and $\widehat{y}$ resulting 
in these oscillations (it could be decreased by decreasing the sampling time or using a higher-order discretization method).

To show the individual estimation performances of the three estimation methods, Fig. 6 shows the dc-offset $e_{0}:=y_{0}-\widehat{y}_{0}$ and the fundamental estimation error $e_{1}:=y_{1}-\widehat{y}_{1}$ which contribute equally to the overall estimation error $e_{y}:=y-\widehat{y}=$ $e_{0}+e_{1}$. Since the $\mathbf{S F A O} \mathbf{O}_{\emptyset, n}$ is not capable of detecting the dcoffset, the respective $e_{0}$ signal is not shown in the first subplot. However, it is still capable of estimating the fundamental signal properly. In conclusion, the $\mathbf{m F A O}_{0, n}$ has the best estimation performance overall.

\section{B. Discussion of Scenario (S2)}

Scenario (S2) considers a signal with ten harmonics (including fundamental) plus dc-offset. It has a known fundamental frequency and undergoes step-like signal parameter changes in dc-offset, harmonic amplitudes and phase angles (see above). $\mathbf{m F A O}_{0, n}$ and $\mathbf{S F A O} \boldsymbol{\emptyset}_{\emptyset, n}$ are now implemented with ten mSOGIs and sSOGIs (i.e. $n=10$ ), respectively. The $\mathbf{s F A O}_{0,1}$ still comes with only one sSOGI. For all methods, the FLLs are turned off (as for Scenario (S1)), since the frequency is assumed to be known (i.e. $\widehat{f}=f$ ).

The results for this scenario are illustrated in Fig. 7 where the individual subplots show input $y(---)$, estimate $\widehat{y}$ and estimation error $e_{y}=y-\widehat{y}$ of $\mathbf{S F A O} \mathbf{O}_{0,1}(-), \mathbf{s F A O}_{\emptyset, n}(-)$ and $\mathbf{m F A O}_{0, n}(-)$, respectively. Due to the parallelization of the mSOGIs, the $\mathbf{m F A O}_{0, n}$ is capable of tracking the input within a few milliseconds whereas the $\mathbf{s F A O} \mathbf{O}_{\emptyset, n}$ with parallelized sSOGIs exhibits a non-zero estimation error resulting from the missing dc-offset estimation. Since the $\mathbf{S F A \mathbf { O } _ { 0 , 1 }}$ contains only one sSOGI, it is not capable of estimating the input signal correctly. In view of the harmonic content, the error resulting from the sampling issue is significantly higher for all estimation methods.

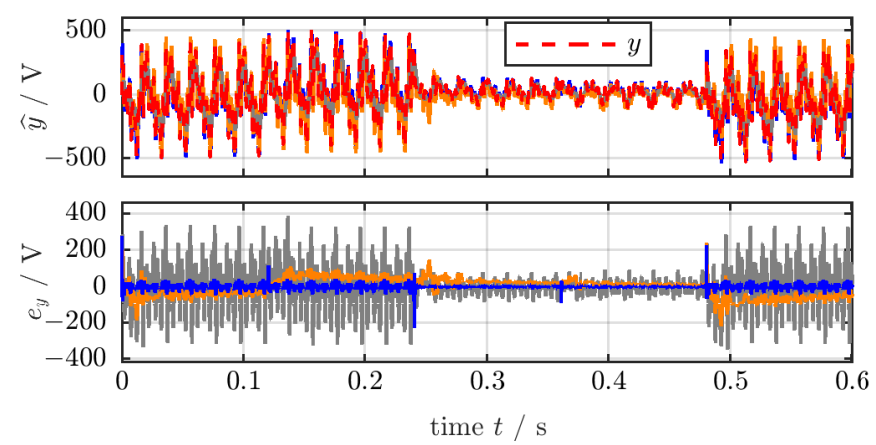

Figure 7: Measurement results for Scenario (S2) (signal with dc-offset, harmonics and known frequency): The signals shown are input $y(--)$, estimate $\widehat{y}$ and estimation error $e_{y}=y-\widehat{y}$ of $\mathbf{s F A O}_{0,1}(-)$, $\mathbf{S F A O}_{\emptyset, n}(-)$ and $\mathbf{m F A O}_{0, n}(-)$, respectively.

Fig. 8 shows the individual estimation errors $e_{0}, e_{1}, \ldots$, $e_{10}$ of dc-offset and harmonics estimation. Note that $\mathbf{s} \mathbf{F A O} \mathbf{O}_{0,1}$ detects the dc-offset precisely; whereas the fundamental error component is affected by all other harmonic estimation errors as those are not estimated at all. As for Scenario (S1), the $\mathbf{S F A O} \mathbf{O}_{\emptyset, n}$ estimates the harmonic content correctly but, clearly, the dc-offset estimation is missing. The $\mathbf{m F A O}_{0, n}$ again provides the best estimation performance concerning
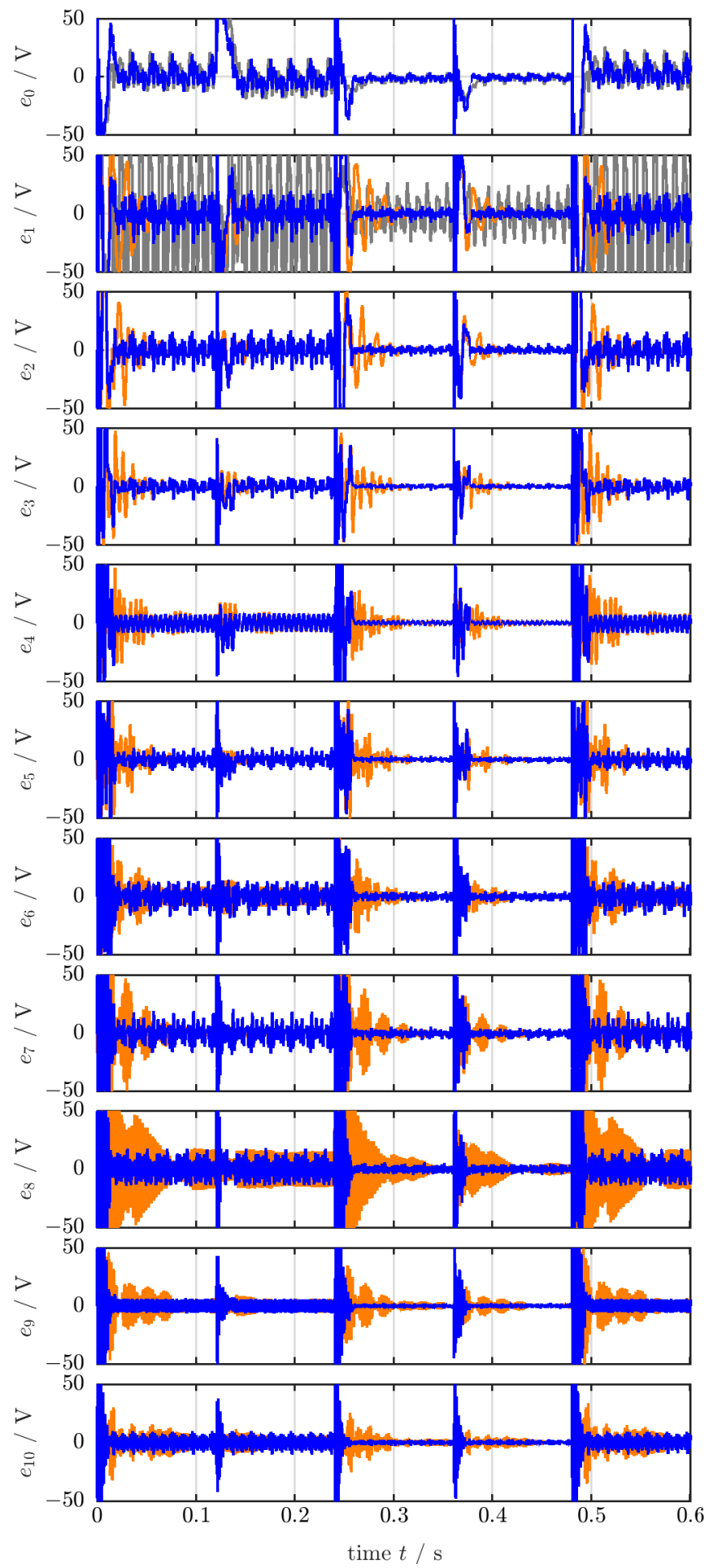

Figure 8: Zoomed-in estimation errors of dc-offset and harmonic signals for Scenario (S2) (signal with dc-offset, harmonics and known frequency): The signals shown are estimation error $e_{0}=y_{0}-\widehat{y}_{0}$ and $e_{\nu}=y_{\nu}-\widehat{y}_{\nu}$ for all $\nu \in\{1, \ldots, 10\}$ of $\mathbf{s F A O}_{0,1}(-)$, sFAO $_{\emptyset, n}(\stackrel{\nu}{\longrightarrow})$ and mFAO $_{0, n}(\longleftarrow)$, respectively.

estimation speed and accuracy - overall and in each individual signal component, respectively.

\section{Discussion of Scenario (S3)}

For Scenario (S3), the considered signal contains a dc-offset and a fundamental component. It now comes with varying 
and unknown angular frequency. The signal undergoes steplike changes in frequency, phase angle and amplitude; in particular, note the interval with zero fundamental component. For all three estimation methods, the FLLs are now activated to achieve frequency adaption as well.

In Fig. 9, input $y(---)$ and its respective estimates $\widehat{y}$ of $\mathbf{S F A O}_{0,1}(-), \mathbf{S F A O}_{\emptyset, n}(-)$ and $\mathbf{m F A O}_{0, n}(-)$ are shown in the first subplot. The second and third subplot show estimation error $e_{y}$, frequency $f \&$ its estimate $\widehat{f}$ of all three methods, respectively. All estimation errors $e_{y}$ are only slightly affected by the frequency adaptions in the FLLs; especially the settling times do not differ too much from those of Scenario (S1). The speeds of the frequency adaption by sFLL and mFLL are almost identical with $60 \mathrm{~ms}$. However, due to the dc-offset, the $\mathbf{S F A O} \mathbf{O}_{\emptyset, n}$ without the capability of dc-offset detection fails to estimate the frequency correctly (oscillations around the correct frequency occur). At $t=0,24 \mathrm{~s}$, when the amplitude sag of $-75 \%$ occurs, the mFLL of the $\mathbf{m F A O}_{0, n}$ does not overshoot like the sFLLs of $\mathbf{s F A O}_{0,1}$ and $\mathbf{S F A O} \mathbf{O}_{\emptyset, n}$. During the interval $0,36 \mathrm{~s} \leq t<0,48 \mathrm{~s}$, when there is no ac component in $y$, the input signal does not contain any frequency information anymore (which is visualized by a missing reference). Hence, all frequency estimators try to track a non-existing reference. For $_{\mathbf{S F A O}} \mathbf{O O}_{0,1}(-)$ and $\mathbf{s F A O} \mathbf{\emptyset}_{\emptyset, n}(-)$ with sFLL, this leads to frequency estimations tending to zero, where the sFLLs are locked and cannot recover even if the fundamental signal comes back within the interval $0,48 \mathrm{~s} \leq t<0,60 \mathrm{~s}$. Due to $\mathrm{AW}$, the frequency estimate of the mFLL is held within the predefined frequency interval $\left[\omega_{\min }, \omega_{\max }\right]$ and does not tend to zero. When the ac component is present again, the mFLL is able to recover and to track the frequency correctly.
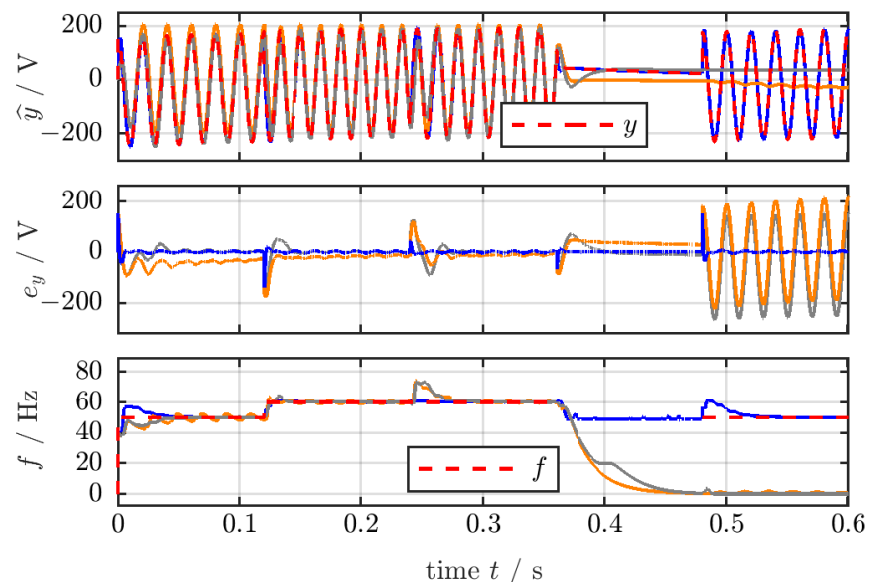

Figure 9: Measurement results for Scenario (S3) (fundamental signal with dc-offset and unknown frequency): The signals shown are input $y$ (- - ), estimate $\widehat{y}$, estimation error $e_{y}=y-\widehat{y}$, frequency $f$ and its estimate $\widehat{f}$ of $\mathbf{S F A O}_{0,1}(-), \mathbf{s F A O}_{\emptyset, n}(-)$ and $\mathbf{m F A O}_{0, n}$ (-), respectively.

In Fig. 10, the individual estimation errors $e_{0}$ and $e_{1}$ for Scenario (S3) are shown. It can be seen that, also for this case when the FLLs are turned on, the proposed $\mathbf{m F A O}_{0, n}(-)$ is the fastest method overall and for each individual signal component.

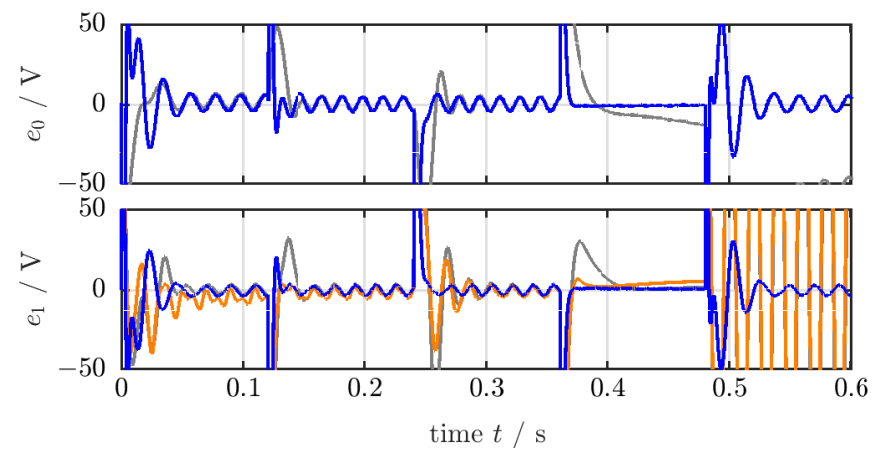

Figure 10: Zoomed-in estimation errors of dc-offset and harmonic signal for Scenario (S3) (fundamental signal with dc-offset and unknown frequency): The signals shown are estimation errors $e_{0}=y_{0}-\widehat{y}_{0}$ and $e_{1}=y_{1}-\widehat{y}_{1}$ of $\mathbf{s F A O}_{0,1}(-), \mathbf{S F A O}_{\emptyset, n}(\stackrel{\square}{\square}$ and $\mathbf{m F A O}_{0, n}(-)$, respectively.

\section{Discussion of Scenario (S4)}

For Scenario (S4), a signal consisting of fundamental, nine harmonics and dc-offset is considered. It has a varying (angular) fundamental frequency and jumps in frequency, harmonic phase angles and amplitudes. Again, for all three methods, the FLLs are turned on.

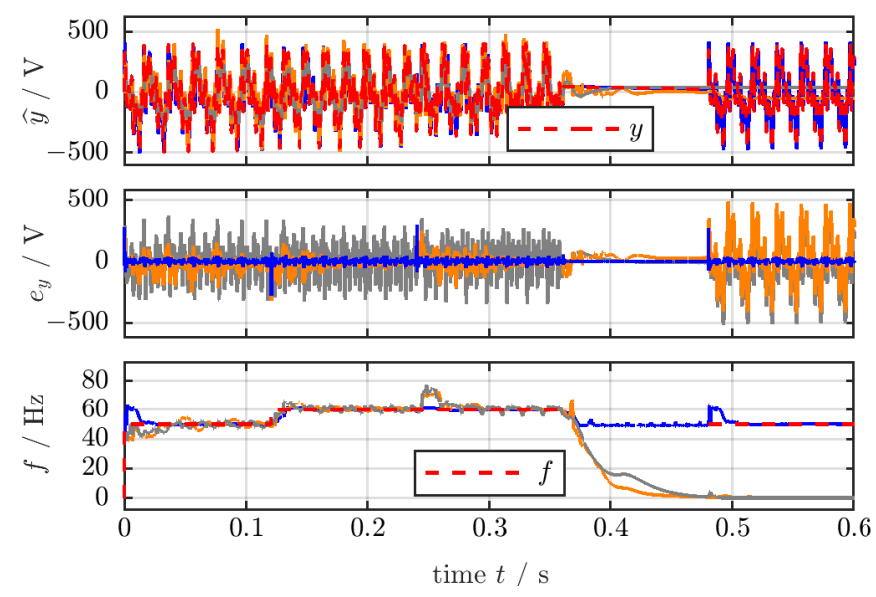

Figure 11: Measurement results for Scenario (S4) (signal with dc-offset, harmonics and unknown frequency): The signals shown are input $y(-=)$ and the estimates $\widehat{y}$, the respective estimation errors $e_{y}=y-\widehat{y}$, frequency $f$ and the respective estimates $\widehat{f}$ of $\mathbf{S F A O} \mathbf{O}_{0,1}$

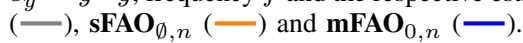

Fig. 11 depicts input $y(---)$ and its respective estimates $\widehat{y}$ of $\mathbf{S F A O}_{0,1}(\square), \mathbf{S F A O}_{\emptyset, n}(-)$ and $\mathbf{m F A O}_{0, n}(-)$ in the first subplot and the respective estimation errors $e_{y}$ in the second subplot (with identical color code). In the third subplot, actual frequency $f$ and its estimates $\widehat{f}$ are plotted. Again, the settling times of the input estimation errors are not significantly influenced by the FLLs. But, since the $\mathbf{S F A O}_{0,1}$ (-) is not designed to estimate harmonics, it fails to estimate the input properly leading to significant ripples in $e_{y}$. Similar results with large ripples in $e_{y}$ are obtained by the $\mathbf{S F A O} \mathbf{O}_{\emptyset, n}$ (-). Moreover, both methods are not able to estimate the frequency correctly until $t \leq 0,36 \mathrm{~s}$ (oscillations are present); after $t>0,36 \mathrm{~s}$, both sFLLs fail completely and are not able to recover when the ac components with frequency information come back (similar malfunctioning as already 


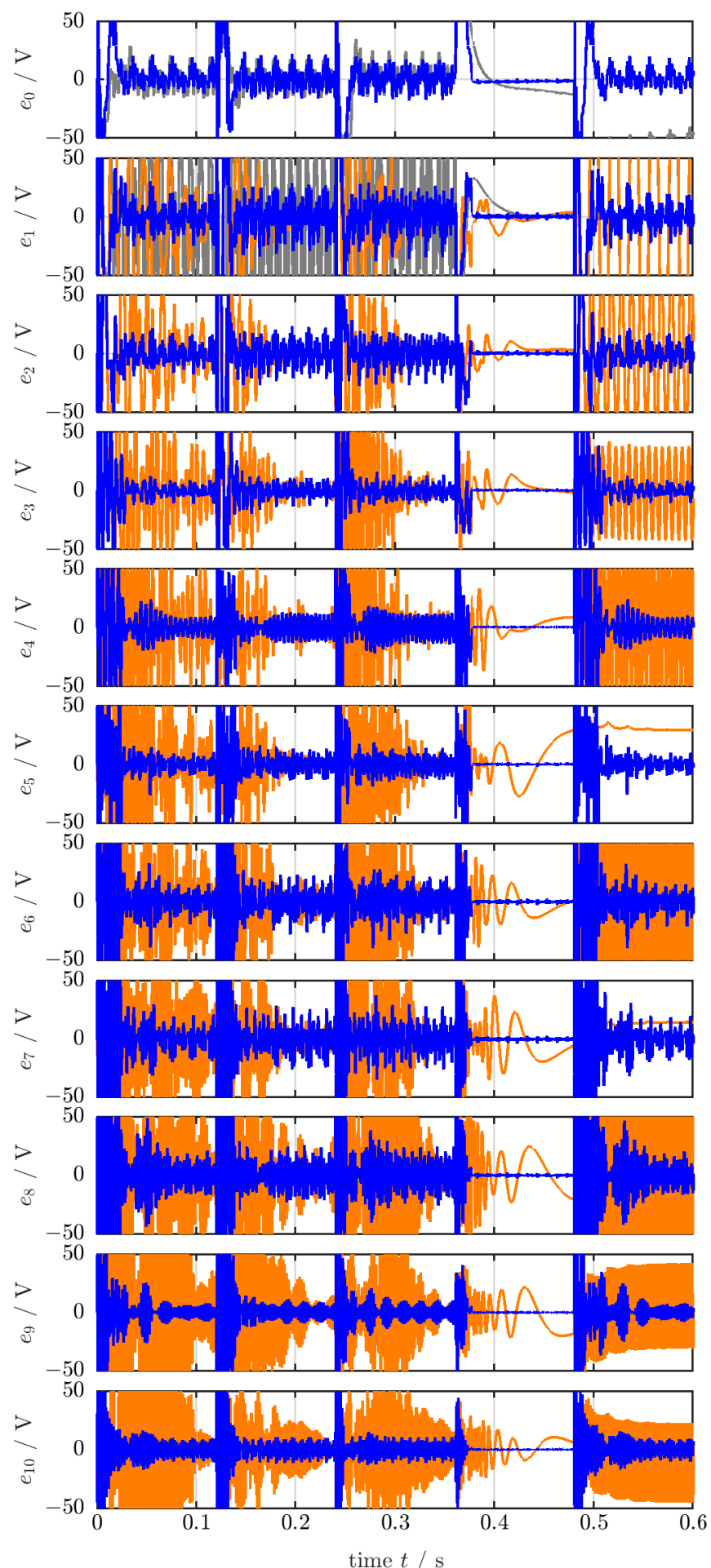

Figure 12: Zoomed-in estimation errors of dc-offset and harmonic signals for Scenario (S4) (signal with dc-offset, harmonics and unknown frequency): The signals shown are the respective estimation errors $e_{0}=y_{0}-\widehat{y}_{0}$ and $e_{\nu}=y_{\nu}-\widehat{y}_{\nu}$ with $\nu \in\{1, \ldots, 10\}$ for $\mathbf{S F A O}_{0,1}(\longleftarrow), \mathbf{s F A O}_{\emptyset, n}(\stackrel{\nu}{\longrightarrow})$ and $\mathbf{m F A O}_{0, n}(-)$.

observed in Scenario (S3)). In contrast to those, the estimation performance of the $\mathbf{m F A O}_{0, n}(-)$ is still acceptable within the interval $t \in[0,36 \mathrm{~s}, 0,48 \mathrm{~s})$ and very good for the remaining time intervals. A similar good performance of the $\mathbf{m F A O}_{0, n}$ (-) can be observed in Fig. 12 for the individual dc-offset $e_{0}$ and harmonic estimation errors $e_{1}, \ldots, e_{10}$. Nevertheless, noise sensitivity of the $\mathbf{m F A O}_{0, n}$ is rather obvious but less than that of $\mathbf{S F A O}_{0,1}(-)$ and $\mathbf{S F A O} \emptyset_{\emptyset, n}(-)$, which both are not capable of achieving an acceptable estimation at all.

\section{CONCLUSION AND OUTLOOK}

A Frequency Adaptive Observer (FAO) - consisting of DC-intergrator (DCI) and parallelized modified Second-Order Generalized Integrators (mSOGIs) and a modified FrequencyLocked Loop (mFLL) with Gain Normalization (GN), signcorrect Anti-Windup (AW) decision function, Rate Limitation (RL) and Low-Pass Filters (LPFs) - has been proposed. It constitutes a unified method to estimate dc-offset, fundamental and harmonic components and (angular) frequency of arbitrarily distorted single-phase signals in grids with dc-offset. Hereby, the recently reported mSOGIs which allow for a (theoretically and) arbitrarily fast tuning of the individual harmonic estimations were used. The key observation for the design of the FAO was the observability property of the underlying signal generation system (internal model) which led to a simple and analytical tuning rule by pole placement. The effectiveness and improved estimation performance of the proposed FAO with and without mFLL was shown by extensive measurement results which were compared to available estimation methods in literature. In future contributions, the following three main goals shall be achieved: (i) Estimation of arbitrary harmonic content without knowing the explicit harmonic orders, (ii) improvement of the frequency adaption to achieve significantly faster estimation speeds and (iii) application of the proposed FAO to three-phase systems to extract symmetrical components of all harmonic components (i.e. to extend the results in [20]).

\section{REFERENCES}

[1] K. R. Patil and H. H. Patel, "Modified dual second-order generalised integrator fll for synchronization of a distributed generator to a weak grid," in 2016 IEEE 16th International Conference on Environment and Electrical Engineering (EEEIC), pp. 1-5, June 2016.

[2] G. Fedele, A. Ferrise, and D. Frascino, "Structural properties of the sogi system for parameters estimation of a biased sinusoid," in 2010 9th International Conference on Environment and Electrical Engineering, pp. 438-441, May 2010.

[3] G. Zhu, Q. Yuan, and X. Yang, "Research and analysis of sogiqsg integral saturation in the application of grid synchronization," in 2019 IEEE International Conference on Mechatronics and Automation (ICMA), pp. 1167-1171, Aug 2019.

[4] D. Janik, J. Talla, T. Komrska, and Z. Peroutka, "Optimalization of sogi pll for single-phase converter control systems: Second order generalized integrator (sogi)," in 2013 International Conference on Applied Electronics, pp. 1-4, Sept 2013.

[5] C. M. Hackl and M. Landerer, "Modified second-order generalized integrators with modified frequency locked loop for fast harmonics estimation of distorted single-phase signals," IEEE Transactions on Power Electronics, 2019.

[6] S. Golestan, J. M. Guerrero, J. C. Vasquez, A. M. Abusorrah, and Y. Al-Turki, "Modeling, tuning, and performance comparison of secondorder-generalized-integrator-based flls," IEEE Transactions on Power Electronics, vol. 33, pp. 10229-10239, Dec 2018.

[7] J. S. Park, D. C. Lee, and T. L. Van, "Advanced single-phase sogi-fll using self-tuning gain based on fuzzy logic," in 2013 IEEE ECCE Asia Downunder, pp. 1282-1288, June 2013.

[8] A. E. Karkevandi and M. J. Daryani, "Frequency estimation with antiwindup to improve sogi filter transient response to voltage sags," in 2018 6th International Istanbul Smart Grids and Cities Congress and Fair (ICSG), pp. 188-192, April 2018. 
9] T. D. C. Busarello, K. Zeb, A. Peres, V. S. R. V. Oruganti, and M. G. Simoes, "Designing a second order generalized integrator digital phase locked loop based on a frequency response approach," in 2019 IEEE PES Innovative Smart Grid Technologies Conference - Latin America (ISGT Latin America), pp. 1-6, Sep. 2019.

[10] C. Xie, K. Li, J. Zou, K. Zhou, and J. M. Guerrero, "Multiple secondorder generalized integrators based comb filter for fast selective harmonic extraction," in 2019 IEEE Applied Power Electronics Conference and Exposition (APEC), pp. 2427-2432, March 2019.

[11] Z. Xin, Z. Qin, M. Lu, P. C. Loh, and F. Blaabjerg, "A new secondorder generalized integrator based quadrature signal generator with enhanced performance," in 2016 IEEE Energy Conversion Congress and Exposition (ECCE), pp. 1-7, Sept 2016.

[12] M. Mojiri, M. Karimi-Ghartemani, and A. Bakhshai, "Time-domain signal analysis using adaptive notch filter," IEEE Transactions on Signal Processing, vol. 55, pp. 85-93, Jan 2007.

[13] M. R. Krstić, S. Lubura, S. Lale, M. Šoja, M. Ikić, and D. Milovanović, "Analysis of discretization methods applied on dc-sogi block as part of srf-pll structure," in 2016 International Symposium on Industrial Electronics (INDEL), pp. 1-5, Nov 2016.

[14] B. Hoepfner and R. Vick, "Symmetrical components detection with ffdsogi-pll under distorted grid conditions," in 2019 International Conference on Smart Energy Systems and Technologies (SEST), pp. 1-6, Sep. 2019.

[15] F. Xiao, L. Dong, L. Li, and X. Liao, "A frequency-fixed sogi-based pll for single-phase grid-connected converters," IEEE Transactions on Power Electronics, vol. 32, pp. 1713-1719, March 2017.

[16] X. He, H. Geng, and G. Yang, "Reinvestigation of single-phase flls," IEEE Access, vol. 7, pp. 13178-13188, 2019.

[17] S. Golestan, J. M. Guerrero, F. Musavi, and J. Vasquez, "Single-phase frequency-locked loops: A comprehensive review," IEEE Transactions on Power Electronics, pp. 1-1, 2019.

[18] P. Rodriguez, A. Luna, I. Candela, R. Mujal, R. Teodorescu, and F. Blaabjerg, "Multiresonant frequency-locked loop for grid synchronization of power converters under distorted grid conditions," IEEE Transactions on Industrial Electronics, vol. 58, pp. 127-138, Jan 2011.

[19] F. Muzi and M. Barbati, "A real-time harmonic monitoring aimed at improving smart grid power quality," in 2011 IEEE International Conference on Smart Measurements of Future Grids (SMFG) Proceedings, pp. 95-100, Nov 2011

[20] C. M. Hackl and M. Landerer, "A unified method for online detection of phase variables and symmetrical components of unbalanced three-phase systems with harmonic distortion," Energies, vol. 12, no. 17, 2019.

[21] Z. Dai, W. Lin, and H. Lin, "Estimation of single-phase grid voltage parameters with zero steady-state error," IEEE Transactions on Power Electronics, vol. 31, pp. 3867-3879, May 2016.

[22] M. Karimi-Ghartemani, S. A. Khajehoddin, P. K. Jain, A. Bakhshai, and M. Mojiri, "Addressing DC component in PLL and notch filter algorithms," IEEE Transactions on Power Electronics, vol. 27, pp. 7886, Jan 2012.

[23] M. Xie, H. Wen, C. Zhu, and Y. Yang "Dc offset rejection improvement in single-phase sogi-pll algorithms: Methods review and experimental evaluation," IEEE Access, vol. 5, pp. 12810-12819, 2017.

[24] Z. Yan, H. He, J. Li, M. Su, and C. Zhang, "Double fundamental frequency PLL with second order generalized integrator under unbalanced grid voltages," in 2014 International Power Electronics and Application Conference and Exposition, pp. 108-113, Nov 2014.

[25] H. K. Yada and M. S. R. Murthy, "An improved control algorithm for dstatcom based on single-phase sogi-pll under varying load conditions and adverse grid conditions," in 2016 IEEE International Conference on Power Electronics, Drives and Energy Systems (PEDES), pp. 1-6, Dec 2016.

[26] T. Ngo, Q. Nguyen, and S. Santoso, "Improving performance of singlephase sogi-fll under dc-offset voltage condition," in IECON 2014 - 40th Annual Conference of the IEEE Industrial Electronics Society, pp. 15371541, Oct 2014

[27] H. Yi, X. Wang, F. Blaabjerg, and F. Zhuo, "Impedance analysis of sogi-fll-based grid synchronization," IEEE Transactions on Power Electronics, vol. 32, pp. 7409-7413, Oct 2017.

[28] J. Matas, H. Martin, J. de la Hoz, A. Abusorrah, Y. A. Al-Turki, and M. Al-Hindawi, "A family of gradient descent grid frequency estimators for the sogi filter," IEEE Transactions on Power Electronics, vol. PP, no. 99 , pp. $1-1,2017$

[29] R. Teodorescu, M. Liserre, and P. Rodriguez, Grid Converters for Photovoltaic and Wind Power Systems. Chichester, United Kingdom: John Wiley \& Sons, Ltd., 2011.
[30] W. M. Wonham, Linear Multivariable Control: A Geometric Approach. No. 10 in Applications of Mathematics, Berlin: Springer-Verlag, 3rd ed. 1985.

[31] C. M. Hackl and M. Landerer, "Modified second-order generalized integrators with modified frequency locked loop for fast harmonics estimation of distorted single-phase signals (LONG VERSION)," arXiv e-prints, p. arXiv:1902.04653, Feb 2019. 